\title{
INFLUENCE OF AGE ON KINEMATIC ANALYSIS OF FUNCTIONAL REACH TEST: A CROSS-SECTIONAL STUDY
}

original paper

(c) University School of Physical Education in Wroclaw

DOI: https://doi.org/10.5114/hm.2021.98405

\section{JOSE ANTONIO MERCHÁN-BAEZA ${ }^{1}$, MANUEL GONZÁLEZ-SÁNCHEZ ${ }^{2}$, ANTONIO IGNACIO CUESTA-VARGAS ${ }^{2,3}$}

\author{
${ }^{1}$ Research Group on Methodology, Methods, Models and Outcomes of Health and Social Sciences, Faculty of Health \\ Science and Welfare, Centre for Health and Social Care Research, University of Vic - Central University of Catalonia, \\ Vic, Spain \\ ${ }^{2}$ Universidad de Málaga, Departamento de Fisioterapia, Instituto de Investigación Biomédica de Málaga, Málaga, Spain \\ ${ }^{3}$ School of Clinical Sciences, Faculty of Health, Queensland University of Technology, Brisbane, Australia
}

\begin{abstract}
Purpose. Ageing is associated with deterioration and alterations in physical function, affecting community mobility, balance, skills, or daily living activities. The aim of the study was to kinematically analyse the influence of age on balance in young and older adults, as well as to compare the kinematics of the trunk and lumbar regions during Functional Reach Test (FRT). Methods. The cross-sectional study involved 5 healthy older adults and 6 healthy young adults. Each participant performed FRT instrumentalized with 2 inertial sensors attached in the trunk (T7) and lumbar (L5-S2) regions. Direct (time and displacement) and indirect (velocity and acceleration) kinematic variables, as well as mid-range achieved in FRT were analysed.

Results. The participants' mean age was $73.04 \pm 3.58$ years among the older adults and $23.5 \pm 1.52$ years among the young adults. Significant differences in time (trunk: $-7.61 \pm 2.14 \mathrm{~s}$; lumbar: $-6.40 \pm 1.06 \mathrm{~s}$ ) and displacement (trunk: $16.33 \pm 4.21^{\circ}$ ) were identified; in the lumbar region, no significant displacement differences were observed $(p>0.05)$. Similarly, in the partial intervals analysed during FRT (from starting position to maximum displacement/imbalance, and return to the initial position), significant differences $(p<0.05)$ were revealed in all direct and indirect variables.

Conclusions. When comparing FRT performance in healthy young and elderly subjects from a kinematic point of view, significant differences were found in the vast majority of kinematic variables analysed in both lumbar and trunk region.
\end{abstract}

Key words: age, young adults, older adults, telemetry, movement, postural balance

\section{Introduction}

Ageing is a natural process characterized by a series of biological changes that result in the reduction of muscle mass and strength, as well as loss of mobility and/ or postural stability, affecting both the static and dynamic balance [1]. The maximum optimal control of postural sway is achieved in adolescence and continues until the age of 60 years [1].

The detection, analysis, and gaining knowledge of changes in balance and gait are part of routine clinical practice. Numerous tests have been developed for this purpose, one of the most common being the Functional
Reach Test (FRT) [2]. FRT is used to measure semi-static balance [3]. It is a simple, fast, cheap, accurate, portable, and clinically accepted test to measure balance [2]. FRT is applied to clinically evaluate the anteroposterior balance and to identify the risk of falls among older people [4].

It has been shown that an inertial sensor located at the lower back region can reliably produce data allowing the identification and interpretation of motion [5]. Furthermore, it is a non-invasive, portable, and very accurate tool with high validity, sensitivity, reliability, and specificity [6]. Knowing if it is possible to parameterize FRT with inertial sensors would allow,

Correspondence address: Manuel González-Sánchez, Universidad de Málaga, Departamento de Fisioterapia, Instituto de Investigación Biomédica de Málaga, Arquitecto Francisco Peñalosa, 3, 29071 Málaga, 29071, Málaga, Spain, e-mail:mgsa23@uma.es

Received: October 16, 2019

Accepted for publication: June 1, 2020

Citation: Merchán-Baeza JA, González-Sánchez M, Cuesta-Vargas AI. Influence of age on kinematic analysis of functional reach test: a cross-sectional study. Hum Mov. 2021;22(3):77-83; doi: https://doi.org/10.5114/hm.2021.98405. 


\section{HUMAN MOVEMENT}

J. Merchán-Baeza, M. González-Sánchez, A. Cuesta-Vargas, Influence of age on balance

with objective measurement values, to analyse the impact of age and to more precisely identify the physical and functional aspects that are altered during aging, thus facilitating prevention interventions and lifestyle changes. No studies have been identified that would analyse the execution of FRT from a kinematic point of view by using 2 inertial sensors and comparing healthy young and older adults.

The aim of this study was to kinematically analyse the influence of age on balance in young adults and older adults, as well as to compare the kinematics of the trunk and lumbar regions during the execution of FRT.

\section{Material and methods}

\section{Participants}

The cross-sectional study involved 5 healthy older adults and 6 healthy young adults. The former were recruited from a public health centre in Málaga, the latter from the Faculty of Health Sciences of the University of Málaga. The subjects were compared kinematically during the execution of FRT. They received an information sheet which explained the nature of the study and the process of participation.

The inclusion criteria were the following: age of $20-30$ years or over 60 years and being able to stay standing for more than 30 seconds without external support. Individuals were excluded if having limitations in walking; severe problems with communication; cardiovascular, respiratory, orthopaedic, neurologic, or metabolic diseases; secondary neurological disorders; and/or if failing to provide an informed consent. Version 3.1 of $\mathrm{G}^{*}$ Power software was used to estimate the sample size, with the consideration of sufficient statistical power and an alpha error (0.05).

The younger age group was delimited to ensure that the participants had fully developed their ability for postural sway and the older participants were of an age at which people had usually lost the most optimal control of postural sway [1].

\section{Functional Reach Test}

FRT is a test designed to analyse semi-static balance. Its execution starts from a standing position, the shoulders must be flexed at $90^{\circ}$ (parallel to the ground). From this initial position, the participant is to progressively move forward with their trunk (without moving their feet) while keeping their arms parallel to the ground. Having reached the point where, in order not to fall, they should take a step, the subject returns to the starting position. The measurement obtained from this test is the difference between the start point and the end of the fingers [3] (Figure 1). FRT has a high test-retest reliability $(r: 0.89-0.98)$ [7].

\section{Inertial sensors}

The inertial sensors used are the InertiaCube $3^{\text {TM }}$ model (InterSense Inc., Bedford, MA, USA), working with a sampling frequency of $180 \mathrm{~Hz}$. Each unit features 9 sensors, covering a $360^{\circ}$ scanning motion along the 3 axes (yaw, pitch, and roll), which provides a high
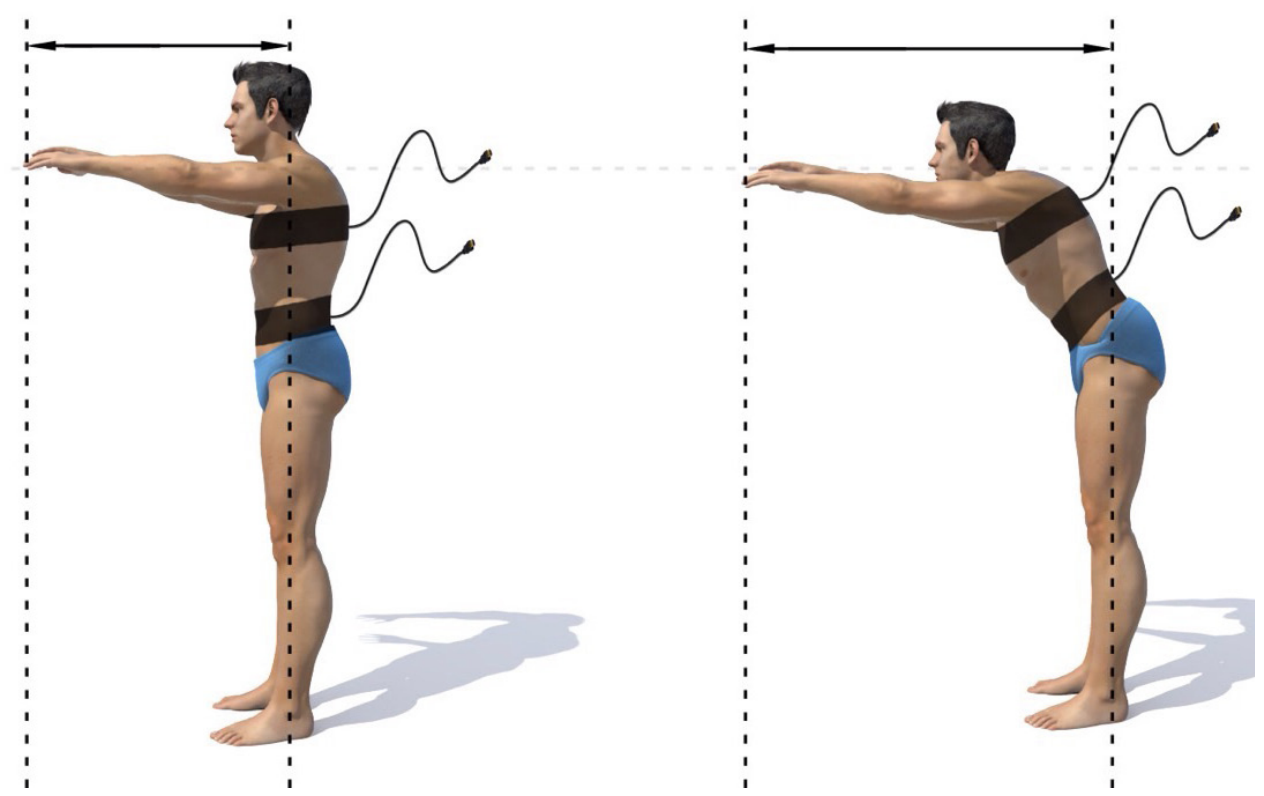

Figure 1. Functional Reach Test execution 
degree of accuracy, sensitivity, and stability, with portability, specificity, validity (0.657-0.998), and reliability (0.84-0.97).

\section{Procedure}

Anthropometric and demographic data were collected through a short questionnaire. The researchers explained how the participants should execute FRT and offered them an opportunity to make a practice attempt. Three executions of FRT were conducted by each subject in order to ensure the reliability of the test. The beginning of the test was adjusted to $0^{\circ}$ by the computer software in the offline analysis.

\section{Outcome measures}

The participants were fitted with a sensor located in the lumbar region (L5-S2) and another one on the trunk (T7) [8]. The inertial sensors were placed so that the origin of the coordinates was positioned in the left posterior-inferior vertex. In the execution of FRT, 3 reference points were established: the starting point (A), the maximum angular displacement (B), and the return to the starting point $(\mathrm{C})$. On this basis, 3 movement intervals were distinguished $(\mathrm{AB}, \mathrm{BC}$, and $\mathrm{AC})$.

\section{Direct variables}

FRT distance was the distance achieved by the participant in the execution of the test. The variables explained below were obtained from the kinematic record collected by the inertial sensors in the pitch axis:

- maximum angular lumbosacral/thoracic FRT displacement: the angular variation of the pitch axis from the start of the test until the maximum reach was attained;

- time of maximum angular lumbosacral/thoracic FRT displacement: the time from the start position to the maximum reach;

- time of return to starting position: the time needed to return from the maximum reach to the starting position;

- total FRT time: the time from the beginning of the movement until the return to the starting position.

\section{Indirect variables}

On the basis of the data previously extracted, the following variables were calculated:

- average FRT velocity: the average velocity at which the participant performed the test;
- maximum angular lumbosacral/thoracic FRT displacement velocity: the average velocity at which the participant moved from the start until they obtained the maximum reach;

- velocity of returning to start position: the average velocity at which the participant returned from the maximum reach to the starting position;

- average FRT acceleration: the average acceleration at which the participant executed the complete test;

- maximum angular lumbosacral/thoracic FRT displacement average acceleration: the mean acceleration at which the participant performed the movement from the starting point to the maximum reach;

- average acceleration of return to FRT starting position: mean acceleration at which the participant returned from the maximum reach to the starting point.

Subsequently, the result of displacement and the maximum and minimum velocity and acceleration of FRT was calculated by the square root of the sum of the squares of the 3 axes. The means and standard deviations of the results previously determined were also established. Furthermore, the means and standard deviations of $\mathrm{X}, \mathrm{Y}$, and $\mathrm{Z}$ were calculated for the maximum, minimum, and mean of the velocity and acceleration in both sensors.

\section{Data analysis}

A descriptive analysis was conducted of the midrange achieved by the participants in the FRT and each of the kinematic variables collected by the inertial sensors. Then, the records of the directly measured variables and the indirect variables were compared between the trunk and lumbar regions. Mann-Whitney $\mathrm{U}$ test was used for nonparametric variables and Student's $t$-test for parametric variables. The index of significance was set at $p<0.05$. In addition, an intergroup comparison was performed of the 2 study groups and between the 2 sensors. The Statistical Package for the Social Sciences (SPSS, version 17.0 for Windows, IL, USA) was applied in the statistical analysis.

\section{Ethical approval}

The research related to human use has complied with all the relevant national regulations and institutional policies, has followed the tenets of the Declaration of Helsinki, and has been approved by the ethics committee of the Faculty of Health Sciences at the University of Málaga. 


\section{Informed consent}

Informed consent has been obtained from all individuals included in this study. The informed consent form explained clearly that their participation was completely voluntary, that they could withdraw from the study at any time, and that their personal data would be protected in accordance with the Organic Law on the Protection of Personal Data 19/55.

\section{Results}

The descriptive characteristics of the healthy young adults were: mean age of $23.5 \pm 1.52$ years, weight of $69.68 \pm 8.95 \mathrm{~kg}$, and height of $168.83 \pm 7.05 \mathrm{~cm}$. The descriptive characteristics of the healthy older group were: mean age of $73.04 \pm 3.58$ years, weight of 72.38 $\pm 11.94 \mathrm{~kg}$, and height of $163.11 \pm 7.02 \mathrm{~cm}$.

The analysis of the kinematic data recorded by the inertial sensors and the comparison between groups shows significant differences between healthy young and healthy older adults in the variables measured by both inertial sensors. The functional reach was higher in the younger group (difference: $3.2, p \leq 0.01$ ). In the 3 FRT intervals, healthy young adults recorded faster motion (trunk AC: $-7.61, p \leq 0.001$; lumbar AC: -6.40 , $p \leq 0.01$ ), higher velocity (trunk AC: $5.76, p \leq 0.01$; lumbar AC: $3.24, p \leq 0.001$ ), greater acceleration (trunk AC: $1.11, p \leq 0.001$; lumbar AC: $0.71, p \leq 0.01$ ), and travers- ing a greater distance (trunk AC: 16.33, $p \leq 0.001$; lumbar AC: 1.76).

Table 1 presents the mean and standard deviation of each of the variables in both groups, as well as the difference obtained in the analysis between the two groups for the trunk sensor. Healthy young adults achieved a greater distance in the functional reach $(39.50 \mathrm{~cm})$ and greater angular displacement in the 3 intervals. This significant difference is reflected in the other measured variables. When analysing the 4 variables measured by the inertial sensor on the trunk, one can observe that healthy young adults achieved a higher angular displacement (difference AC: $16.33^{\circ}$ ), higher velocity (difference AC: $5.76 \%$ s), greater acceleration (difference AC: $1.11 \% \mathrm{~s}^{2}$ ), and a shorter time (difference AC: $-7.61 \mathrm{~s}$ ) than the healthy older adults in the 3 intervals.

Table 2 presents the mean and standard deviation of the variables in the 2 groups and the differences obtained for the lumbar sensor. The values of the 4 measured variables are greater in the healthy older group. The sensor positioned in the lumbar region showed the same pattern as the trunk sensor in the $\mathrm{BC}$ and $\mathrm{AC}$ intervals. However, in the $\mathrm{AB}$ interval, healthy older adults achieved a greater angular displacement (difference: $1.2^{\circ}$ ), higher velocity (difference: $26.87^{\circ} / \mathrm{s}$ ), greater acceleration (difference: $1.72 \% \mathrm{~s}^{2}$ ), and a longer time (difference: $6.72 \mathrm{~s}$ ) than the young group.

Table 1. Description and differences between groups of the kinematic variables of Functional Reach Test measured with the trunk inertial sensor

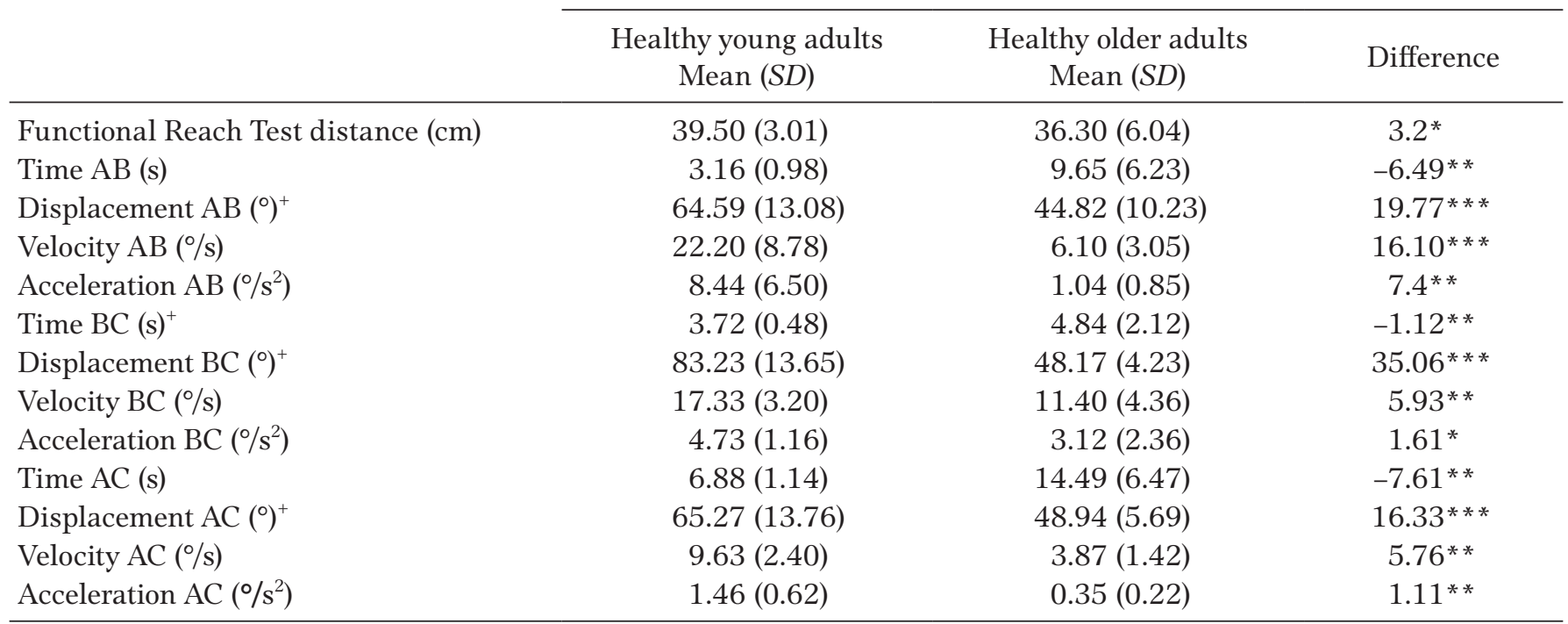

A - FRT beginning, B - maximum angular displacement, $\mathrm{C}$ - return to initial position

${ }^{+}$non-parametric distribution

Significance: ${ }^{*} p \leq 0.05,{ }^{*} p \leq 0.01,{ }^{* *} p \leq 0.001$ 
Table 2. Description and differences between groups of the kinematic variables of Functional Reach Test measured with the lumbar inertial sensor

\begin{tabular}{|c|c|c|c|}
\hline & & & \\
\hline & $\begin{array}{c}\text { Healthy young adults } \\
\text { Mean }(S D)\end{array}$ & $\begin{array}{c}\text { Healthy older adults } \\
\text { Mean }(S D)\end{array}$ & Difference \\
\hline Time AB (s) & 3.47 (1.18) & $9.59(5.12)$ & $-6.12 * * *$ \\
\hline Displacement $\mathrm{AB}\left({ }^{\circ}\right)^{+}$ & $49.87(19.62)$ & $51.07(7.19)$ & -1.2 \\
\hline Velocity AB (\%/s) & $15.61(8.87)$ & $42.48(11.27)$ & $26.87 * * *$ \\
\hline Acceleration $\mathrm{AB}\left(\% / \mathrm{s}^{2}\right)$ & $5.59(5.61)$ & $7.31(5.77)$ & $-1.72 * * *$ \\
\hline Time BC (s) & $3.56(0.49)$ & $4.85(0.86)$ & $-1.29 * *$ \\
\hline Displacement $\mathrm{BC}\left({ }^{\circ}\right)^{+}$ & $49.31(18.87)$ & $48.28(6.42)$ & 1.03 \\
\hline Velocity BC (\%/s) & $13.57(4.51)$ & $10.24(2.37)$ & $3.33^{* *}$ \\
\hline Acceleration $\mathrm{BC}\left(\% / \mathrm{s}^{2}\right)$ & $3.78(1.14)$ & $2.25(1.00)$ & $1.53^{* *}$ \\
\hline Time AC $(s)^{+}$ & $7.04(1.36)$ & $13.44(4.87)$ & $-6.40 * * *$ \\
\hline Displacement $\mathrm{AC}\left({ }^{\circ}\right)^{+}$ & $51.53(18.17)$ & $49.77(9.51)$ & 1.76 \\
\hline Velocity AC (\%/s) & $7.35(2.60)$ & $4.11(1.78)$ & $3.24^{* *}$ \\
\hline Acceleration $\mathrm{AC}\left(\% / \mathrm{s}^{2}\right)$ & $1.08(0.60)$ & $0.37(0.25)$ & $0.71 * *$ \\
\hline
\end{tabular}

A - FRT beginning, B - maximum angular displacement, $\mathrm{C}$ - return to initial position

${ }^{+}$non-parametric distribution

Significance: ${ }^{*} p \leq 0.01,{ }^{* *} p \leq 0.001$

\section{Discussion}

In the presented study, the linear and angular displacement was greater in the healthy young adults than among the healthy older adults. There were no significant differences between the kinematic variables obtained from the inertial sensors in the trunk and lumbar positions (except in some indirect variables). Therefore, the findings confirm the hypothesis that was set in the study a priori.

\section{Differences in kinematic variables}

As expected, significant between-group differences were found in the execution of the test. This allows us to compare the 'normal' pattern of young adults against older adults, and thus to quantify and specify the influence of age on balance. The kinematic record shows that older adults compensate the insecurity they feel with the displacement of their lower back area by exposing the centre of mass outside their limits of balance, which has a direct impact on all activities of their daily lives. These data are consistent with those obtained in previous studies, in which healthy young people presented greater trunk flexion than healthy older people (difference: $8.1^{\circ}$ and $7.4^{\circ}$ ), greater displacement of the centre of pressure (difference: $31 \mathrm{~mm}$ ), and higher linear velocity of centre of mass (difference: $0.05 \mathrm{~m} / \mathrm{s}$ ) [9-11].

This apparent difference in the general pattern of the results between the young adult group and the healthy older adults could be explained as a compensation method applied by the elderly. That is, when they move the arms and trunk forward, they feel insecure because their centre of pressure moves forward and is positioned beyond the limits of balance, so they move the lower back to compensate $[10,11]$.

When analysing the indirect variables calculated subsequently (velocity and acceleration), one can find that the maximum peak of velocity in the anteroposterior movement was higher in the healthy young adults $(58.56 \%$ s) than in the healthy older group $(27.03 \%$ s); however, the maximum acceleration in the same movement was bigger in the older adults $\left(6.17 \% \mathrm{~s}^{2}\right)$ than in the younger group $\left(1.45^{\circ} / \mathrm{s}^{2}\right)$. In a previous study [12], no large differences were observed in the kinematic variables except for the angular acceleration, which was higher in healthy older adults. This could denote a minor postural control in this group [12].

\section{Functional Reach Test comparison}

between the age groups

The mean distance reached in the FRT by the healthy young adults was $39.50 \mathrm{~cm}$, compared with $36.30 \mathrm{~cm}$ among the healthy older adults, representing a difference between the 2 groups of $3.20 \mathrm{~cm}$. This difference, although significant, is not as remarkable as the ones indicated in other studies, e.g. $16.1 \mathrm{~cm}$ [13], $16.84 \mathrm{~cm}$ [14], and $8.1 \mathrm{~cm} \mathrm{[10].} \mathrm{The} \mathrm{small} \mathrm{difference} \mathrm{shown} \mathrm{be-}$ tween the 2 groups in the present study could be due to the active profile of the healthy older participants, 
who reported walking on 3 days per week on average [15].

The difference found in the functional reach between the 2 populations results mainly from the age factor. With more advanced age, the maintenance of postural balance becomes a more difficult task and any movement that involves the centre of pressure shifting outside the boundaries of the support base is a risk [1]. Therefore, in this group, a series of compensatory mechanisms occur to keep the centre of pressure within the limits of stability at the expense of the anteroposterior range [13]. Some of the compensation mechanisms identified by other authors involved co-activation of antagonistic muscles that provided tightening to the joints of the lower limbs, limitation in trunk flexion, and a greater displacement in the lumbar region vs. the trunk, found in the present study [11, 13].

Groups of healthy older adults who executed FRT in other studies achieved functional reaches of $32 \mathrm{~cm}$ [16], $32.11 \mathrm{~cm} \mathrm{[17],} 33.7 \mathrm{~cm} \mathrm{[18],} \mathrm{and} 37.1 \mathrm{~cm}$ [10], which is consistent with the $36.30 \mathrm{~cm}$ obtained in the present study. However, in some studies [13, 14], similar groups (aged 82 and 81.7 years) achieved a reach of 27.33 and $20.9 \mathrm{~cm}$. The differences with reference to the distance reported for the healthy older adults in this study is probably due to the difference in age between the groups in the various studies and the active profile of the healthy older group in the present study [15]. Moreover, the functional reach achieved by the healthy young adults corroborates the distance obtained by other healthy young groups from other studies [10, 13, 14].

\section{Strengths and limitations}

Although efforts have been made to minimize the effect of skin elasticity during the execution of FRT, it is important to consider its influence on data collection. On the other hand, the results and conclusions obtained by analysing the kinematic data represent an advance in the investigation of movement and postural balance in the study population [19].

\section{Conclusions}

There are significant differences in the kinematic variables recorded when comparing healthy young adults with healthy older adults performing FRT. These differences are significant regardless of whether the recording is made in the subjects' thoracic or lumbar area.

\section{Acknowledgments}

The authors are grateful to the volunteers for their participation.

\section{Disclosure statement}

No author has any financial interest or received any financial benefit from this research.

\section{Conflict of interest}

The authors state no conflict of interest.

\section{References}

1. De Vries EA, Caljouw SR, Coppens MJM, Postema K, Verkerke GJ, Lamoth CJC. Differences between young and older adults in the control of weight shifting within the surface of support. PLoS One. 2014;9(6):e98494; doi: 10.1371/journal.pone.0098494.

2. Maranesi E, Ghetti G, Rabini RA, Fioretti S. Functional reach test: movement strategies in diabetic subjects. Gait Posture. 2014;39(1):501-505; doi: 10.1016/j.gaitpost.2013.08.035.

3. Duncan PW, Weiner DK, Chandler J, Studenski S. Functional reach: a new clinical measure of balance. J Gerontol. 1990;45(6):M192-M197; doi: 10.1093/geronj/ 45.6.m192.

4. Costarella M, Monteleone L, Steindler R, Zuccaro SM. Decline of physical and cognitive conditions in the elderly measured through the functional reach test and the mini-mental state examination. Arch Gerontol Geriatr. 2010;50(3):332-337; doi: 10.1016/j.archger.2009.05.013.

5. Little C, Lee JB, James DA, Davison K. An evaluation of inertial sensor technology in the discrimination of human gait. J Sports Sci. 2013;31(12):1312-1318; doi: 10.1080/02640414.2013.779739.

6. Perez-Cruzado D, González-Sánchez M, Cuesta-Vargas AI. Parameterization and reliability of single-leg balance test assessed with inertial sensors in stroke survivors: a cross-sectional study. Biomed Eng Online. 2014;13:127; doi: 10.1186/1475-925X-13-127.

7. Volkman KG, Stergiou N, Stuberg W, Blanke D, Stoner J. Methods to improve the reliability of the functional reach test in children and adolescents with typical development. Pediatr Phys Ther. 2007;19(1):20-27; doi: 10.1097/01. pep.0000247173.14969.5a.

8. Howcroft J, Kofman J, Lemaire ED. Review of fall risk assessment in geriatric populations using inertial sensors. J Neuroeng Rehabil. 2013;10(1):91; doi: 10.1186/ 1743-0003-10-91.

9. Mancini M, Salarian A, Carlson-Kuhta P, Zampieri C, King L, Chiari L, et al. ISway: a sensitive, valid and reliable measure of postural control. J Neuroeng Rehabil. 2012;9(1):59; doi: 10.1186/1743-0003-9-59.

10. Cavanaugh JT, Shinberg M, Ray L, Shipp KM, Kuchibhatla M, Schenkman M. Kinematic characterization of standing reach: comparison of younger vs. older subjects. 
Clin Biomech. 1999;14(4):271-279; doi: 10.1016/S02680033(98)00074-6.

11. Kozak K, Ashton-Miller JA, Alexander NB. The effect of age and movement speed on maximum forward reach from an elevated surface: a study in healthy women. Clin Biomech. 2003;18(3):190-196; doi: 10.1016/s02680033(02)00205-x.

12. Kato T, Yamamoto S, Miyoshi T, Nakazawa K, Masani K, Nozaki D. Anti-phase action between the angular accelerations of trunk and leg is reduced in the elderly. Gait Posture. 2014;40(1):107-112; doi: 10.1016/j.gaitpost.2014.03.006.

13. Nagai K, Yamada M, Uemura K, Yamada Y, Ichihashi N, Tsuboyama T. Differences in muscle coactivation during postural control between healthy older and young adults. Arch Gerontol Geriatr. 2011;53(3):338-343; doi: 10.1016/j.archger.2011.01.003.

14. Norris B, Medley A. The effect of context and age on functional reach performance in healthy adults aged 21 to 94 years. J Geriatr Phys Ther. 2011;34(2):82-87; doi: 10.1519/JPT.0b013e31820aac11.

15. Okubo Y, Osuka Y, Jung S, Rafael F, Tsujimoto T, Aiba T, et al. Walking can be more effective than balance training in fall prevention among community-dwelling older adults. Geriatr Gerontol Int. 2016;16(1):118-125; doi: 10.1111/ggi.12444.

16. Dite W, Temple VA. A clinical test of stepping and change of direction to identify multiple falling older adults. Arch Phys Med Rehabil. 2002;83(11):1566-1571; doi: 10.1053/apmr.2002.35469.

17. Wolf SL, Catlin PA, Gage K, Gurucharri K, Robertson R, Stephen K. Establishing the reliability and validity of measurements of walking time using the Emory Functional Ambulation Profile. Phys Ther. 1999;79(12): 1122-1133; doi: 10.1093/ptj/79.12.1122.

18. Muramoto A, Imagama S, Ito Z, Hirano K, Ishiguro N, Hasegawa Y. Physical performance tests are useful for evaluating and monitoring the severity of locomotive syndrome. J Orthop Sci. 2012;17(6):782-788; doi: 10.1007/s00776-012-0283-z.

19. Liao C-F, Lin S-I. Effects of different movement strategies on forward reach distance. Gait Posture. 2008;28(1): 16-23; doi: 10.1016/j.gaitpost.2007.09.009. 